\title{
EXACT EVALUATION OF SOME HIGHLY OSCILLATORY INTEGRALS
}

\author{
OMRAN KOUBA
}

Abstract. In this note a general result is proved that can be used to evaluate exactly a class of highly oscillatory integrals. The result can also be used to give a procedure to numerically evaluate some oscillatory integrals.

Mathematics subject classification (2010): 11B68, 26A42, 30D10, 33B30, 42B05.

Keywords and phrases: Fourier series, analytic functions, power series expansion, Bernoulli polynomials, polylogarithms, Bessel functions.

\section{REFERENCES}

[1] M. Abramowitz And I. A. Stegan, Handbook of Mathematical Functions, with Formulas, Graphs, and Mathematical Tables, Dover Books on Mathematics, Dover Publication, Inc., New York, (1972).

[2] L. V. Ahlfors, Complex Analysis, third edition, McGraw-Hill, Inc. (1979).

[3] K. B. Athreya And S. N. Lahiri, Measure Theory and Probability Theory, Springer Science+Business Media, LLC. (2006).

[4] C. B. BERndt, The Chief Accountant and Mathematical Friend of Ramanujan - S. Narayana Aiyar, The American Mathematical Monthly, 118 (2011) 767-776, http://dx.doi.org/10.4169/ amer.math.monthly.118.09.767.

[5] F. Bornemann, D. Lauire, S. Wagon and J. Waldvogel, The SIAM 100-Digit Challenge: A Study in High-Accuracy Numerical Computing, Philadelphia, PA: SIAM, (2004).

[6] I. Gradshteyn And I. Ryzhik, Tables of Integrals, Series and Products, 7th ed., Academic Press, (2007).

[7] M. D. Hirchhorn, Problem 11600, The American Mathematical Monthly, 118 (2011) 846, http://dx.doi.org/10.4169/amer.math.monthly.118.09.846.

[8] P. Henrici, Applied and Computational Complex Analysis, Vol. 1, John Wiley, (1974).

[9] Y. KATZnelson, Introduction To Harmonic Analysis, 3rd ed., Cambridge University Press, (2004).

[10] L. LEWIN, Polylogarithms and associated functions, Elsevier North Holland, Inc., (1981).

[11] L. N. Trefethen, The \$100, 100-digit Challenge, SIAM News, 35, No. 6, (2002), 1-3.

[12] G. P. Tolstov and R. A. Silverman, Fourier Series, Dover Books on Mathematics, Dover Publication, Inc., New York, (1962).

[13] J. WALdvogel, Towards a general error theory of the trapezoidal rule, Approximation and Computation, in honor of Gradimir V. Milovanović, W. Gautschi, G. Mastroianni, Th. M. Rassias (eds.), Springer Optimization and its Applications 42, Springer, New York, (2011), pp. 267-282. 